\title{
Confirmation of a new therapeutic option for aggressive or dopamine agonist-resistant prolactin pituitary neuroendocrine tumors
}

\author{
Gerald Raverot ${ }^{1,2,3}$, Alexandre Vasiljevic ${ }^{2,3,4}$, Emmanuel Jouanneau ${ }^{2,3,5}$ and Hélène Lasolle ${ }^{1,2,3}$ \\ ${ }^{1}$ Fédération d'Endocrinologie, Centre de Référence Maladies Rares Hypophysaires (HYPO), Groupement Hospitalier \\ Est, Hospices Civils de Lyon, Bron, France, ${ }^{2}$ Faculté de Médecine Lyon Est, Université Lyon 1, ${ }^{3}$ Inserm U1052, CNRS \\ UMR5286, Cancer Research Centre of Lyon, Lyon, France, ${ }^{4}$ Centre de Pathologie et de Neuropathologie Est, and \\ ${ }^{5}$ Service de Neurochirurgie, Centre de Référence Maladies Rares Hypophysaires (HYPO), Fédération \\ d'Endocrinologie, Groupement Hospitalier Est, Hospices Civils de Lyon, Bron, France
}

Correspondence should be addressed to G Raverot

Email

gerald.raverot@chu-lyon.fr

\begin{abstract}
Recent publications suggested that pasireotide could be a good therapeutic option in some dopamine-resistant or aggressive prolactinomas. We discussed the two published cases and describe another case of poorly differentiated plurihormonal PIT-1-positive adenoma with moderate SSTR2 expression and intense STTR5 expression successfully treated with PAS-LAR $40 \mathrm{mg} / \mathrm{month}$.
\end{abstract}

This very interesting case report by Coopmans et al., demonstrating response to pasireotide therapy in an aggressive dopamine-resistant prolactinoma, recapitulates, in most respects, the description of a previous case published by Lasolle et al. (1). Both these cases demonstrated the rapid and sustained normalization of prolactin secretion in a macroprolactinoma resistant to high doses of cabergoline (up to $7 \mathrm{mg} /$ week). Prolactin normalized within a few weeks ( 2 and 1 month), and the effect was maintained for more than 3 and 9 years respectively. Prolactin normalization was associated with a change of the tumor on MRI in both cases, with higher T2 signal intensity with increasing treatment duration suggestive of cystic degeneration and tumor cell necrosis. However, tumor shrinkage was evident only in the case published here (Coopmans et al.). This discrepancy could be explained by the tumor location, which was limited to the sphenoid sinus in the first published case (1). Indeed, it is frequently observed in macroprolactinoma that despite major tumor shrinkage of the suprasellar extension on cabergoline treatment, shrinkage is less obvious in the sphenoid component of the tumor due to its anatomical characteristics (2).
Contrary to this case in which cabergoline was maintained at a low dose despite cardiac valvular regurgitation, in the Lasolle et al. case (1), cabergoline, being ineffective, was stopped after 3 months of PAS-LAR without an increase in prolactin. Moreover, we were able to reduce the dose of PAS-LAR to $20 \mathrm{mg}$ every 6 weeks associated with slight, clinically non-significant, prolactin increase and without change seen on MRI.

SSTR2 and SSTR5 expression, analyzed by immunohistochemistry, demonstrated variable membranous expression with IRS 2 and 9 for SSTR2a and 4 and 12 for SSTR5 (Lasolle et al. and Coopmans et al. respectively). SSTR2a being low in one case and 10-months treatment with SRL lanreotide autogel at $120 \mathrm{mg} / \mathrm{month}$, a SSTR2a analog, having no effect on prolactin secretion or tumor size, suggested that PAS-LAR action was mediated by SSTR5 expression. IRS for SSTR5 was moderate or high, suggesting that SSTR5 expression analysis could predict tumor response to PAS-LAR and may be used to select patients who may benefit from this treatment before considering temozolomide treatment.

However, it should be noted that SSTR2a and SSTR5 expression are uncommon in prolactinomas. In our cohort

Published by Bioscientifica Ltd. 
of 47 macroprolactinomas, operated in our referral center from 2010 to 2019, SSTR2 and SSTR5 expression were present in $3 / 23$ and $3 / 21$ analyzed tumors respectively. One tumor expressed both receptors, while 4 other tumors expressed SSTR2a or SSTR5 only. IRS for SSTR5 were mild $(3 ; n=1)$ to intense $(12 ; n=2)$.

In addition to our previously published case, we recently treated a 55-year-old woman, who was referred to our institution with a diagnosis of giant silent prolactinoma resistant to cabergoline, with PAS-LAR $40 \mathrm{mg} / \mathrm{month}$. The initial diagnosis was made in another center in June 2016 on MRI performed to evaluate recent visual field defects and severeheadache (Fig. 1). Sheunderwent surgical biopsy which led to the diagnosis of silent prolactinoma. Her prolactin level was normal at diagnosis but according to the pathology report she was treated with cabergoline at a dose of up to $3.5 \mathrm{mg} /$ week. In the absence of a tumor effect, after 6 months of treatment, she was referred to our reference center for the indication of surgical debulking. Surgery via a transcranial approach was performed in December 2016. As expected, post-operative MRI revealed a large residual tumor with persistent optic nerve compression. Pathology demonstrated a PIT-1 positive pituitary neuroendocrine tumor with low expression of prolactin, scattered $\beta$-TSH-positive cells, moderate SSTR2 expression and intense STTR5 expression in 90\% of cells (Fig. 2). The WHO 2017 classification recognizes this subtype of pituitary neuroendocrine tumors as a plurihormonal PIT-1-positive adenoma. These tumors are considered by the $\mathrm{WHO}$ as high-risk pituitary adenomas due to their clinical aggressive behavior (3). PAS-LAR 40 mg/ month was proposed to the patient after a multidisciplinary discussion. The treatment was initiated in May 2017 and was well tolerated without digestive or metabolic complaints. Clinical and MRI evaluation after 4 months of treatment demonstrated rapid ophthalmologic improvement and tumor shrinkage, allowing the continuation of PAS-LAR for an additional 3 months. After 7 months, the residual tumor being stable, transsphenoidal endoscopic surgery was proposed to allow a better debulking and raise the possibility of adjuvant radiotherapy. MRI performed 3 months after the second surgery demonstrated good tumor removal of the intrasellar part, but the large extrasellar extension close to the optic chiasm was unchanged. The second pathology report demonstrated high STTR5 expression and thus PAS-
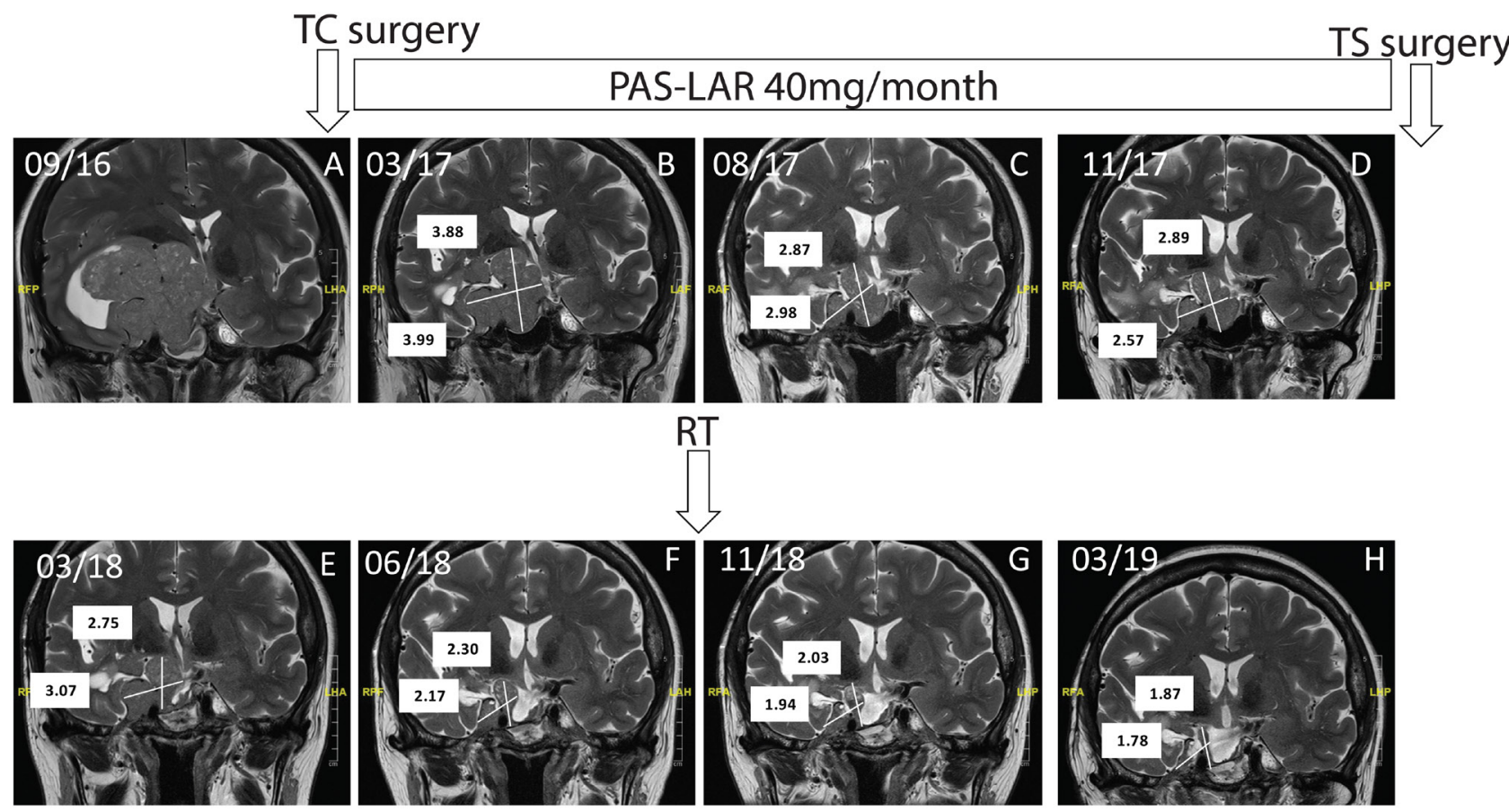

PAS-LAR 40mg/month

\section{Figure 1}

MRI scans during the different surgical and medical therapies. T2-weight MRI scan obtained after 6 months of cabergoline before transcranial (TC) surgery (A), 3 months post-op and pre-PAS-LAR (B), 4 months (C) and 7 months post-PAS-LAR before transsphenoidal (TS) surgery (D), 3 months post-surgery and before re-initiation of PAS-LAR (E), and during 3-9 and 12 months of PAS-LAR ( $F, G$ and $H$ ) and radiation therapy (RT). 

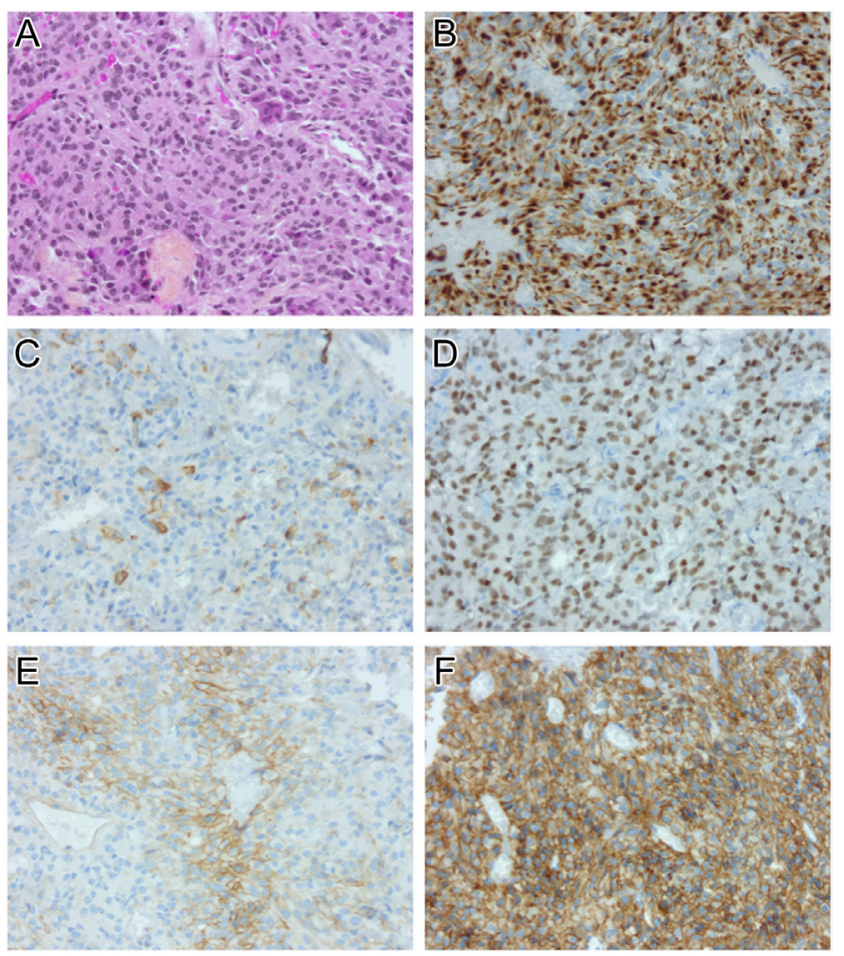

\section{Figure 2}

Histopathological features of the tumor at first surgery. (A) The tumor is composed of diffusely arranged cells showing weakly eosinophilic cytoplasm. Nuclei are slightly irregular with a thin chromatin and a prominent eosinophilic nucleolus (HematoxylinPhloxine-Saffron staining). (B) Cytokeratin 18 immunopositivity in tumoral cells shows a perinuclear and Golgi distribution. Fibrous bodies are rare. (C) Prolactin immunopositivity present in $8 \%$ of tumoral cells. (D) There is a diffuse nuclear immuno-expression of PIT-1 in neoplastic cells. (E) 20\% of tumoral cells express SSTR2 with a moderate intensity (IRS $=4$ ). (F) $90 \%$ of tumoral cells express SSTR5 with a strong intensity (IRS =12). (Original magnification $\times 200$ ); IRS, immunoreactive score. There were no significant features of proliferation observed (no mitotic figures, Ki67 <3\%, P53 negative).

LAR $40 \mathrm{mg} / \mathrm{month}$ was proposed after multidisciplinary discussion. This second course of PAS-LAR was associated with additional significant tumor shrinkage, after 3 months, allowing the continuation of the treatment. Independent of this tumor shrinkage, as decided after the first multidisciplinary discussion, stereotactic radiotherapy (54Gy) was performed according to the ESE guideline on aggressive pituitary tumors (4). At last follow-up after
12 months of PAS-LAR $40 \mathrm{mg} / \mathrm{month}$ and 4 months after radiation therapy, the residual tumor size continued to reduce. Of note, T2 signal changes were visible only after 12 months of PAS-LAR.

The encouraging results of these two papers and our personal experience led us to consider PAS-LAR treatment before temozolomide treatment in dopamine-resistant prolactinomas and aggressive pituitary tumors. Indeed, temozolomide can control aggressive prolactinomas in about $40 \%$ of cases but long-term control is not guaranteed, and side effects are more frequent compared to PAS-LAR (5). However, no data are available on PAS-LAR for treating prolactin carcinomas with proven metastasis, so in such rare conditions, temozolomide is still the firstline option. Moreover, SSTR5 expression being rare in prolactinoma $(<15 \%)$, its analysis should be considered before starting PAS-LAR in this indication.

\section{Declaration of interest}

Gerald Raverot received investigator-initiated research grants from Novartis and Ipsen, speaker's fees from Ipsen Pharma, Novartis Pharma, Pfizer Inc. and consulting fees from Novartis. Emmanuel Jouanneau received speaker's fees from Novartis Pharma and Baxter. The other authors have nothing to disclose.

\section{Funding}

This research did not receive any specific grant from any funding agency in the public, commercial or not-for-profit sector.

\section{References}

1 Lasolle H, Vasiljevic A, Borson-Chazot F \& Raverot G. Pasireotide: A potential therapeutic alternative for resistant prolactinoma. Annales d'Endocrinologie 201980 84-88. (https://doi.org/10.1016/j. ando.2018.07.013)

2 Delgrange E, Duprez T \& Maiter D. Influence of parasellar extension of macroprolactinomas defined by magnetic resonance imaging on their responsiveness to dopamine agonist therapy. Clinical Endocrinology 200664 456-462. (https://doi.org/10.1111/j.13652265.2006.02493.x)

3 Lloyd RV, Osamura RY, Klöppel G \& Rosai J. Pathology and Genetics of Tumours of Endocrine Organs. World Health Organization Classification of Tumours, vol. 4th. Lyon: IARC Press, 2017.

4 Raverot G, Burman P, McCormack A, Heaney A, Petersenn S, Popovic V, Trouillas J, Dekkers OM \& European Society of Endocrinology. European Society of Endocrinology Clinical Practice Guidelines for the management of aggressive pituitary tumours and carcinomas. European Journal of Endocrinology 2018178 G1-G24. (https://doi.org/10.1530/EJE-17-0796)

5 Lasolle H, Cortet C, Castinetti F, Cloix L, Caron P, Delemer B, Desailloud R, Jublanc C, Lebrun-Frenay C, Sadoul JL et al. Temozolomide treatment can improve overall survival in aggressive pituitary tumors and pituitary carcinomas. European Journal of Endocrinology 2017 176 769-777. (https://doi.org/10.1530/EJE-16-0979) 